\title{
Análisis del valor comunicativo de las redes sociales en el ámbito universitario: estudio de los usos de Twitter en el aula
}

\author{
Eva García Montero \\ Universidad Camilo José Cela \\ egarmo@ucjc.edu \\ Marián De la Morena TABOAdA \\ Universidad Camilo José Cela \\ mmorena@ucjc.edu \\ Laura Melendo RodríGuez-CARMONA \\ Universidad Camilo José Cela \\ lmelendo@ucjc.edu
}

Recibido: 25/07/2012

Aceptado: 16/10/2012

\begin{abstract}
Resumen
El objetivo de este artículo es presentar el proyecto iniciado por la Facultad de Comunicación en el curso 2011-2012 con el propósito de convertir Twitter en un punto de encuentro entre profesores y alumnos fuera del ámbito universitario. Este estudio pretende analizar y monitorizar el uso que han dado docentes y alumnos a Twitter en sus clases. El objetivo no es establecer valores cuantitativos sino marcar algunas tendencias y subrayar posibles hipótesis que se puedan contrastar con estudios posteriores. Entre ellas destaca saber si las redes sociales pueden convertirse en una herramienta docente fuera del aula tradicional. Para ello se realizará un repaso de las redes sociales en el entorno educativo y se analizará Twitter como red social y como herramienta docente.
\end{abstract}

Palabras clave: redes sociales, universidad, comunicación, Twitter, docentes, alumnos

\section{Analysis of the communicative value of the social networks in the university scope: study of the uses of Twitter in the classroom}

\begin{abstract}
The aim of this article is to present a project started by the Faculty of Communication in 2011-2012 to make Twitter a meeting point for professors and students outside the academic environment. The study analyzes and monitors the use of Twitter in class by a group of students and faculty members. The objective is not to establish quantitative data but to trace certain uses and underline possible hypotheses to be tested in future studies. Among these hypotheses one outstands from the rest: to know if social networks can become a useful educational tool outside the traditional classroom environment. A review of the uses of social networks in the learning environment and a more specific analysis of Twitter as social network and learning tool are included.
\end{abstract}

Key words: social networks, university, communication, Twitter, teachers, students

\section{Referencia normalizada}

GARCÍA MONTERO, Eva; DE LA MORENA TABOADA, Marián y MELENDO RODRÍGUEZ-CARMONA, Laura (2012): "análisis del valor comunicativo de las redes sociales en el ámbito universitario: estudio de los usos de twitter en el aula". Estudios sobre el mensaje periodístico. Vol. 18, núm. especial octubre, págs.: 393-403. Madrid, Servicio de Publicaciones de la Universidad Complutense.

Sumario: 1. Introducción. 2. Metodología. 3. Redes sociales: comunicación y educación; 3.1. Twitter; 3.2. Microblogging; 3.3. Prácticas de uso. 4. Resultados del estudio y conclusiones. 5. Referencias bibliográficas. 


\section{Introducción}

Las implicaciones pedagógicas del uso de las redes sociales en la enseñanza y el aprendizaje son muy variadas. Si usamos las redes sociales adecuadamente en el aula podemos establecer nuevas vías de comunicación y abrir nuevos canales para encuentros formativos que además aportan una dinámica distinta al día a día de la docencia.

Es interesante destacar que las redes sociales permiten a los alumnos determinar y controlar su proceso de aprendizaje a la vez que reflexionan sobre el mismo potenciando estrategias metacognitivas que permiten a los alumnos ser mucho más conscientes de los recursos y herramientas que les facilitarán este proceso de adquisición de conocimiento. De esta manera hacen frente a nuevas situaciones de aprendizaje en las que se potencia el trabajo en equipo así como otras estrategias afectivas y sociales que juegan un papel determinante en contextos comunicativos. El trabajo con redes sociales refuerza las relaciones entre los miembros del grupo de clase y se puede aprovechar esta relación de confianza que se establece para proporcionar un entorno libre de estrés. En esta línea, Hossain y Wigand (2004) señalan que las relaciones de confianza que se establecen entre los participantes son de gran importancia en el proceso educativo en tanto que proceso integral.

En términos generales podemos decir que el proceso de enseñar con redes sociales no es una mera transmisión de datos sino que se convierte en un ejercicio colaborativo de compilación, orquestación e integración de información en construcción de conocimiento (Educause, 2009).

Esta orquestación favorece el establecimiento de sinergias para un aprendizaje continuo que se extenderá en el tiempo, es decir que las redes sociales favorecen y ayudan a configurar el Entorno Personal de Aprendizaje de las personas (Castañeda y Gutierrez, 2010).

El Entorno Personal de Aprendizaje no está limitado espacialmente ya que las redes sociales permiten colaborar con profesores y grupos de alumnos situados en cualquier lugar del mundo que estén interesados en las mismas áreas o proyectos y participar activamente en proyectos conjuntos de creación de conocimiento. Recordando a Lamarck podemos concluir esta introducción diciendo que si la evolución está basada en una interacción constructiva y cooperativa de los organismos para adaptarse a los cambios de un entorno dinámico las redes sociales son unos excelentes facilitadores de esta re-evolución.

\section{Metodología}

Este estudio trata de determinar el uso que se le ha dado a la cuenta de Twitter @UCJCComunicacion, cuenta oficial del grupo de primero de comunicación de la UCJC. El principal objetivo es conocer la finalidad con la que se usa la cuenta de Twitter del grupo de Primero de Comunicación de la Facultad de Ciencias de la Comunicación de la UCJC. En cuanto a la metodología se analizó el tipo de contenido de todos los mensajes emitidos desde la cuenta de Twitter mencionada en la fase cualitativa y posteriormente se procedió a catalogar los 447 tweets emitidos. Las catego- 
rías de uso que se han empleado son fruto de la interpretación de las autoras de los establecidos por Marc Cortés ${ }^{1}$ :

1. Compartir información

2. Transmitir noticias y eventos en directo

3. Generar conversación

4. Ocio

5. Exhibicionismo y medio de mantener el contacto

Universo: 447 tweets enviados desde el 18 de enero hasta el 21 de junio de 2012. Fechas de trabajo de campo: julio 2012.

\section{Redes Sociales: comunicación y educación}

Sabemos que el universo es relacional. La influencia de las conexiones sociales es una evidencia a lo largo de la historia que está jalonada de ejemplos en los que una buena red de contactos junto con una adecuada estrategia de comunicación ha lanzado a la fama a ciertos científicos o escritores y condenado al olvido a otros; véanse los casos de Charles Darwin y Alfred Russel Wallace o Henry James y Mary Wilkins Freeman por citar algún ejemplo.

La actividad docente se desarrolla habitualmente en grupos de alumnos cada uno de los cuales dispone de un profesor para una materia determinada. Esta estructura docente se asemeja al modelo que representan las redes sociales que se caracterizan por su falta de especialización en un área o actividad específica. El valor de las redes sociales radica fundamentalmente en su capacidad para conectar a las personas entre sí permitiendo conexiones entre un número ilimitado de personas a diferencia del correo electrónico y otras herramientas de comunicación virtual que tienen límite de usuarios.

Con el advenimiento de las nuevas tendencias pedagógicas auspiciadas por el Espacio Europeo de Educación Superior (EEES) se han incorporado a la docencia universitaria nuevas plataformas tecnológicas que posibilitan la interacción del docente con el alumno fuera de la tradicional clase magistral.

Así, las redes sociales se han introducido en el aula o, quizá, lo que ha ocurrido es que el docente se ha introducido en las redes sociales buscando una mayor interacción con los alumnos.

\subsection{Twitter}

En estos momentos de máxima exposición a la información en el que se han gestado importantes movimientos sociales al amparo de los "me gusta" y los "re-tweet" y "opinar en directo" se ha transformado en el hilo conductor de la economía y los negocios no podemos dar la espalda a la incorporación de estos elementos al ámbito académico. Esta incorporación podemos hacerla desde una doble perspectiva, siguiendo la información que generan líderes de opinión en la materia y produciendo materiales propios y originales dentro del grupo educativo.

${ }^{1}$ Fuente: elaboración propia y Marc Cortés en "Nanoblogging: Los usos de las nuevas plataformas de comunicación en la red" 
Si hace unos años alguien nos hubiese preguntado si encontraríamos interesante una red social en la que pudiéramos informar lo que estamos haciendo en cada momento, las respuestas hubieran sido de lo más variadas, pero casi con total seguridad todas ellas redundarían en que sería una verdadera estupidez dejar descubierta nuestra intimidad de forma tan gratuita y sin gracia.

Twitter ${ }^{2}$ es una red de información en tiempo real que conecta a los usuarios a las últimas noticias sobre aquello que consideran interesante. Simplemente a través de conectarse a los flujos que elijan y siguiendo las conversaciones. Según el informe de resultados de la $4^{\mathrm{a}}$ oleada del Observatorio de Redes Sociales que realiza The Cocktail Analysis se detecta sobre Twitter una carga emocional más intensa y positiva que sobre ninguna otra red. Los usuarios hablan sobre lo que pasa en esta red social, que en si misma se ha convertido en un objeto conversacional, invita a participar e involucrarse, está viva, es dinámica.

El corazón de Twitter son pequeñas piezas de información, denominadas Tweets. Cada tweet tiene 140 caracteres de longitud. Asociado a cada tweet hay un panel con detalles que facilitan información adicional, contenido en profundidad y multimedia. Puedes contar tu historia dentro del tweet, o puedes usarlo como titular e incluir información adicional a través de imágenes, videos y otros contenidos multimedia.

Desde su nacimiento esta red social basada en el envío de mensajes cortos de 140 caracteres, ha tenido un crecimiento vertiginoso consiguiendo las siguientes cifras:

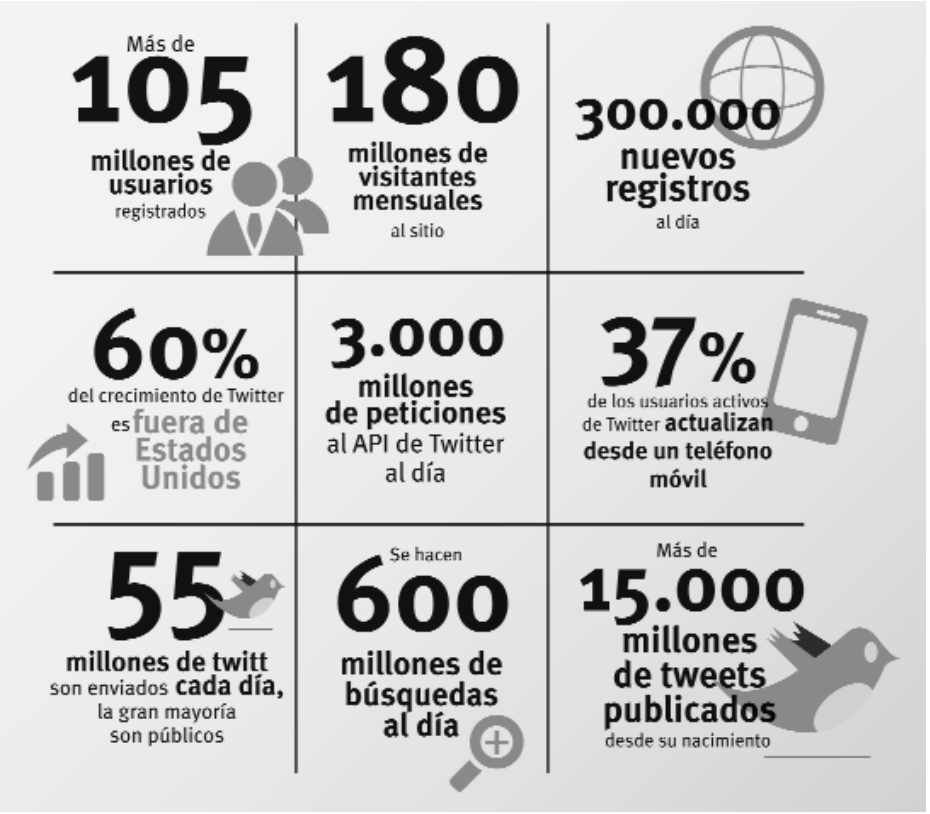

Imagen 1. Cifras del uso de Twitter Fuente: http://www.abc.es/20100531/medios-redesweb/crecimiento-twitter-201005311328.html

${ }^{2}$ La siguiente información es una traducción adaptada de la descripción que Twitter hace de la herramienta y de la aplicación para empresas en su página web. 
Si la idea de este microblogging fue concebida en 2006, será en 2007 cuando la herramienta esté lista para ser lanzada al mercado. A pesar de que enseguida comenzó a ganar adeptos, el crecimiento de su base de usuarios ha sido lento en comparación con otras redes. Sin embargo, a partir de 2009 ha tenido un crecimiento exponencial en muchos países como consecuencia de la propagación del boca a boca por parte de los usuarios y medios de comunicación y al factor de haber sido traducida paulatinamente a otros idiomas, entre ellos el español (noviembre de 2009).

El crecimiento de Twitter ha sido espectacular en los últimos dos años. A mediados de abril de 2010 Biz Stone, co-fundador de Twitter, anunciaba en su página personal que la herramienta contaba con 105 millones de usuarios registrados y se generaban 55 millones de tweets por día, contando con un crecimiento de 300.000 nuevos usuarios diarios.

En el caso de España no se trata de una de las herramientas favoritas por parte de los usuarios de redes sociales como lo son Facebook y Tuenti. Sin embargo podemos afirmar que del año 2009 a la actualidad, es un caso de éxito por la capacidad de asentamiento y desarrollo que ha tenido en nuestro país. Siguiendo el informe sobre Twitter llevado a cabo por Adigital ${ }^{3}$, encontramos el llamativo dato de que un 54,8\% de los usuarios españoles se dieron de alta en Twitter durante los dos últimos años. Entre estos usuarios un $50,70 \%$ son hombres, frente a un $49,30 \%$ de mujeres. En cuanto a la distribución por edades la mayoría de los usuarios $(42,40 \%)$ tienen entre 35-44 años mientras que en el 2010 la mayoría tenían entre 25-34 años. Otra característica propia de esta herramienta es que desde 2009 ha experimentado una clara evolución cualitativa de usuarios y su uso se ha profesionalizado: el $80,8 \%$ de los usuarios tiene estudios superiores; el $60,65 \%$ posee una profesión relacionada en alguna medida con Internet, siendo uno de cada cuatro usuarios trabajador por cuenta propia; el $26,5 \%$ de los tweets publicados se decantan netamente hacia la temática profesional antes que hacia la personal (solo el 9,03\%), aunque el 21,5\% de los usuarios afirma que acostumbra a publicar ambos tipos de tweets. El uso de Twister por parte de los universitarios queda reducido a un $11,5 \%$. La mayoría de los usuarios $(80,8 \%)$ son postuniversitarios.

El objetivo más importante a la hora de usar Twitter es "informarse de lo que sucede en el mundo online" ( $83,9 \%$ de los usuarios) seguido por "mejorar a nivel profesional" $(56,5 \%)$.

Respecto a los contenidos de los tweets, los tweets más publicados son aquellos en los que se comparten noticias y estudios a nivel profesional $(77,7 \%)$ y enlaces en general $(76,4 \%)$. Los menos publicados: el 67,20 de los usuarios nunca han publicado ofertas de trabajo, ni promocionando entradas en su blog $(53,1 \%)$ y tampoco ha comunicado sus estados de ánimo (51,7\%). Un $63,4 \%$ de los usuarios recomienda productos y un $61,9 \%$, comparte quejas acerca de un producto o servicio.

El 71,8\% de los usuarios acceden a Twitter varias veces al día y casi el 59\% de los usuarios publican al menos un tweet al día. Además, se dispara el uso de dispositivos

${ }^{3}$ Asociación Española de la Economía Digital (Mayo 2012), Estudio de uso de Twitter en España. 
móviles para conectarse a Twitter. E1 70\% de los usuarios asegura que se conecta a través de su smartphone.

En cuanto al futuro de Twitter los usuarios declaran que adquirirá más relevancia como canal de comunicación (62,3\%) y de información (59,4\%).

Para entender mejor qué es Twitter es importante definir qué es en esencia el Microblogging.

\subsection{Microblogging}

Nos referimos como microblogging a una forma de comunicación que consiste en el envío de mensajes cortos de texto a través de herramientas diseñadas especialmente para esta función.

En el caso de Twitter, el servicio más utilizado, el número máximo de caracteres es de 140. Para poder utilizar estas plataformas no es necesario instalar en el ordenador ningún software, ya que los mensajes son escritos, enviados y leídos desde una página web. El acceso es sencillo y abierto a todos, ya que tan solo hay que darse de alta con un e-mail y contraseña en la página web del servicio. Una vez estamos registrados, ya podemos comenzar a enviar mensajes a través de la página y leer los mensajes que otros usuarios han escrito.

Su objetivo es explicar qué se está haciendo en un momento determinado, compartir información con otros usuarios, ofrecer enlaces interesantes a páginas web o, según lo que hemos estado viendo durante los últimos tiempos, contar lo que está pasando en un lugar y tiempo concretos en primera persona. Genís Roca afirma en su página personal que el microblogging puede definirse a partir de la suma de cuatro conceptos:

1. Blog: se escribe como un diario personal o un espacio propio del autor en el que se publican contenidos en orden cronológico.

2. Mensajería instantánea: se trata de sistemas de comunicación que nos permiten mantener conversaciones en tiempo real en Internet con otros usuarios.

3. SMS: se basa en el envío de mensajes de texto de hasta 140 caracteres, que pueden incluir enlaces.

4. Red social: el envío de mensajes se hace a un espacio público que podrá ser leído por todas las personas que nos "sigan", por lo que se produce una expansión de este mensaje.

Existen múltiples plataformas de microblogging que comparten funcionalidades muy similares, que las caracterizan y definen como tal. Sin embargo, es conveniente distinguir dos tipos de plataformas en función del usuario al que se dirigen.

- Herramientas de microblogging externas. El usuario utiliza la plataforma como herramienta de comunicación externa. Estas plataformas no están restringidas a un entorno determinado (a pesar de que el usuario puede proteger sus mensajes). El ejemplo más exitoso y conocido es Twitter.

- Herramientas de microblogging internas. En un principio, estas plataformas se plantearon como una herramienta para gestionar la comunicación interna de las empresas, pero actualmente se han abierto a otro tipo de grupos. Son platafor- 
mas, por lo tanto, que se caracterizan por ser herramientas de comunicación interna entre los miembros de determinado grupo. El ejemplo más conocido es Yammer.

Analizando cómo los usuarios de Twitter utilizan la herramienta, podemos distinguir varios usos de la misma ${ }^{4}$.

- Compartir información: Los usuarios comparten noticias, curiosidades, datos de interés... sobre temas concretos. Twitter se ha convertido para muchos en una fuente primaria para obtener información de calidad gracias al contenido que comparten los usuarios, algunos de ellos expertos en determinadas materias.

- Transmitir noticias y eventos en directo: La inmediatez de difusión de las herramientas y la posibilidad de utilizarlas desde dispositivos móviles, permiten que el usuario pueda comunicar de forma inmediata lo que está pasando en determinado lugar y momento, generando un flujo de información desde diferentes puntos de vista. Hemos podido ver el poder de este uso en catástrofes naturales como el terremoto de Haití o las revueltas en el mundo árabe. Desde una perspectiva totalmente diferente al ejemplo anterior, cada vez también es más corriente que los usuarios comenten en directo grandes eventos sociales, como la gala de los Goya o los Oscar.

- Generar conversación: A partir de un hecho relevante para el usuario se busca generar una conversación, algo que es posible gracias a las menciones (funcionalidad que explicaremos más adelante en este documento) Los usuarios buscan conocer la opinión de otros usuarios con llamadas a la acción, o demandan respuestas a los implicados (por ejemplo, cuando un usuario se dirige a la cuenta de una empresa para preguntar una duda o dejar una queja).

- Ocio: Los usuarios no sólo comparten información útil, sino también links de vídeos o canciones, juegos, chistes... convirtiendo estas herramientas no sólo en una fuente de información, sino también de entretenimiento.

- Exhibicionismo y medio de mantener el contacto: Los usuarios comparten también en estas plataformas pensamientos sueltos, información personal sobre sí mismos, tal y como se hace en otros medios sociales como Facebook. Muchos usuarios lo utilizan también para mantener el contacto con personas de su entorno cercano o lejano y con otros usuarios.

\subsection{Prácticas de uso}

Según el informe de resultados de la $3^{\mathrm{a}}$ oleada del Observatorio de Redes Sociales que realiza The Cocktail Analysis la interacción en las redes sociales es fácil, exige escaso esfuerzo al usuario y es entretenida. La clave para una correcta utilización de Twitter es fomentar la interacción. Este objetivo se consigue mediante diversas estrategias entre las que destacan la capacidad para generar y mantener una conversación, el fomento de la participación y la implicación de los públicos, así como la aportación de contenidos relevantes. Es importante cuidar la actualización pues una de las ven-

${ }^{4}$ Fuente: elaboración propia y Marc Cortés en "Nanoblogging: Los usos de las nuevas plataformas de comunicación en la red" 
tajas del medio es que prima la "noción de tiempo real" por lo tanto la respuesta debe ser rápida. Según un estudio del AIB citado por José Manuel Noguera Vivo, Josep Martínez Polo y María del Mar Grandío Pérez en 2011, los jóvenes son los que mas mensajes públicos y privados envían a través de las redes. Estos nativos digitales se encuentran cómodos compartiendo de forma abierta.

Entre las buenas prácticas que se recomiendan ${ }^{5}$ para el uso de esta herramienta están:

- Seguir a quien te sigue

- Responder de forma frecuente y sincera

- Dotar de humanidad nuestra cuenta

- No hablar solo de uno mismo

- Controlar el número de tweets

- Ser agradecido

- Y por supuesto, cuidar la gramática, la ortografía y la puntación

Diversas investigaciones han puesto de manifiesto cómo el uso frecuente de aplicaciones informáticas ha incrementado algunas funciones cognitivas entre niños y adolescentes. Tardieu y Gyselinck (2003), por ejemplo, pusieron de manifiesto que las rutinas informáticas son fundamentalmente visuales, nuestra memoria de trabajo a pesar de que es más visual que auditiva puede verse sobrecargada por el desorden de estímulos visuales y por tanto resultar menos efectiva mientras nos enfrentamos a sistemas multimedia. Parece que la era digital está afectando a nuestros procesos cognitivos, en algunos casos desarrollando algunas funciones y limitando otras.

\section{Resultados del estudio y conclusiones}

Con este mensaje se inauguraba la cuenta de Twitter@UCJCComunicacion el 18 de enero de 2012 con el fin de tener un punto de encuentro del grupo. Desde esa fecha hasta el 21 de junio la cuenta estuvo activa, con un total de 447 tweets. La cuenta tiene 51 seguidores y sigue a 47 .

Tabla 1. Tipos de usos en Twitter de @UCJCComunicacion. Fuente: elaboración propia con datos del usuario @UCJCComunicacion

\begin{tabular}{lc} 
USO & \% Tweets \\
\hline Compartir información & $43,9 \%$ \\
Exhibicionismo y medio de mantener el contacto & $33,9 \%$ \\
Generar conversación & $5,9 \%$ \\
Ocio & $4,5 \%$ \\
Transmitir noticias y eventos en directo & $11,8 \%$ \\
\hline Total & $100 \%$ \\
\hline
\end{tabular}

${ }_{5}^{5}$ Elaboración propia a partir de información del libro de Juan Diego Polo: Twitter... para quien no usa twitter 


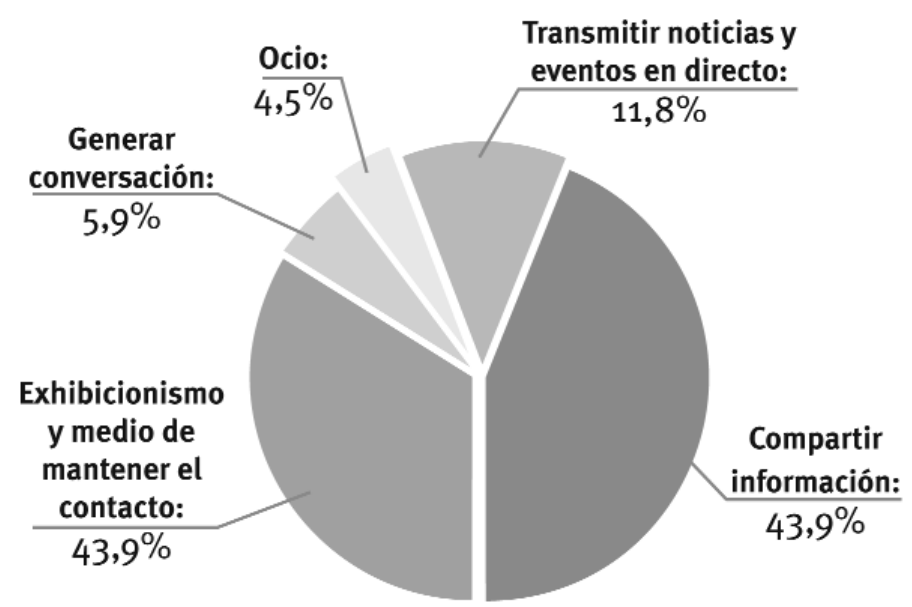

Gráfico 1: Tipos de usos en Twitter de @UCJCComunicacion Fuente: elaboración propia con datos del usuario@UCJCComunicacion

El principal uso que se le ha dado a la cuenta es compartir información, ya que ha sido el objetivo del $43,9 \%$ de los mensajes escritos por el perfil analizado. Los alumnos han compartido noticias, curiosidades, datos de interés... sobre temas relacionados con la marcha del grupo, los horarios de clase, dudas sobre entregas de trabajo, entre otros.

Entre los principales temas, se encuentran informar o recordar fechas importantes para el grupo, asuntos significativos para la propia organización del mismo, recordatorio de tareas o transmitir mensajes de los profesores.

El exhibicionismo y el objetivo de mantener el contacto es el segundo motivo por el que se ha usado la cuenta, con el 33,9\% de los mismos. Por ejemplo saludarse, mantenerse localizados o simplemente compartir algún pensamiento sueltos e información personal sobre sí mismos, tal y como se hace en otros medios sociales como Facebook.

Entre este tipo de mensajes, se encuentran los que desean cohesionar el grupo, en los que simplemente se saluda al grupo. También se emplea para ubicar al grupo dentro de las instalaciones del campus. Y hay otros cuyo fin es el de generar buen ambiente.

Para transmitir noticias y eventos en directo solamente se han escrito el $11,8 \%$, ocupando este uso el tercer puesto en el ranking. Extraña el escaso uso que se le da, lo poco que se aprovecha la inmediatez de difusión de esta herramienta y la posibilidad de utilizarlas desde dispositivos móviles. Pero ha permitido generar un flujo de información para que el grupo pueda comunicarse de forma inmediata, para saber lo que está pasando en determinado lugar y momento.

En cuarto lugar queda el uso de esta herramienta para generar conversación, tan solo empleada en el 5,9\% de los mensajes. Pocas veces han sido las que, a partir de un hecho relevante para el grupo, se ha buscado generar una conversación. 
Dentro de este tipo de uso, se han analizado el sujeto con el que se generaba la conversación. En la mayoría de los casos la conversación se mantenía con un profesor $(42,3 \%)$, en segundo lugar con un alumno del grupo $(38,5 \%)$, en tercer lugar con la Universidad $(3,8 \%)$. El 15,4\% restante de las conversaciones se dirigían a la totalidad de los seguidores de la cuenta.

En último lugar quedan los usos centrados en temas de ocio. Tan sólo el 4,5\% de los mensajes tenían fines de ocio. Los usuarios no sólo comparten información útil, sino también links de vídeos o canciones, juegos, chistes... convirtiendo estas herramientas no sólo en una fuente de información, sino también de entretenimiento.

La red de redes como un lugar donde el individuo escondía su personalidad es una visión anticuada, ya superada, de una Internet de finales de los años noventa, de un primer momento en el que percibíamos sus chats, sus mundos virtuales, como juego, como mundo paralelo, como teatro (diría Goffman), un lugar donde escapar del control social y, por tanto, lugar seguro en el que podíamos experimentar roles o identidades ficticias. No es así en la web social actual, definida incluso, como sociedad de la transparencia. La red resulta un lugar donde el yo, el individuo puede incluso aprehender y compartir conocimiento.

\section{Referencias Bibliográficas}

ASOCIACIÓN ESPAÑOLA DE LA ECONOMÍA DIGITAL (Mayo 2012): Estudio de uso de Twitter en España.

CASTAÑEDA, Linda y GUTIÉRREZ, Isabel (2010): "Redes sociales y otros tejidos online para conectar personas". Aprendizaje con redes sociales. Sevilla, Eduforma

CORTÉS, Marc (2009): Nanoblogging: Los usos de las nuevas plataformas de comunicación en la red. Barcelona, UOC.

EDUCAUSE: "7 Things you should know about Personal Learning Environments". http://net.educause.edu/ir/library/pdf/ELI7049.pdf

HOSSAIN, Liaquat y WIGAND, Rolf T. (2004): "Ict enabled virtual collaboration through trust". Journal of Computer-mediated Communication 10, $\mathrm{n}^{\mathrm{o}} 1$.

NOGUERA VIVO, Josep Manuel; MARTINEZ POLO, Josep; y GRANDÍO PEREZ, María del Mar (2011): Redes Sociales para estudiantes de comunicación. Barcelona, Editorial UOC.

POLO, Juan Diego (2009): Twitter ... para quien no usa Twitter. Libro digital disponible en: www.bubok.com

TARDIEU, Hubert y GYSELINCK, Válérie (2003): "Working memory Constraints in the Integration and Comprehension of Information in a Multimedia Context". Cognition in a digital world. (Ed. van Oostendorp) New Jersey, Lawrence Erlbaum Associates, Inc., Publishers 


\section{Eva GARCÍA MONTERO}

Directora Grado de Publicidad y Relaciones Públicas UCJC egarmo@ucjc.edu

\section{Marián DE LA MORENA TABOADA}

Profesora del Grado de Publicidad y Relaciones Públicas UCJC mmorena@ucjc.edu

\section{Laura MELENDO RODRÍGUEZ-CARMONA}

Profesora del Grado de Publicidad y Relaciones Públicas UCJC lmelendo@ucjc.edu 